\title{
Immune reaction characteristics and the mechanism of anergy induced by recombinant enterotoxin a of Staphylococcus aureus
}

\author{
Shang Wu ${ }^{1,2^{*}}$, Renli Zhang ${ }^{1 *}$, Dana Huang ${ }^{1}$, Yijie Geng ${ }^{1}$, Shitong Gao ${ }^{1}$, Xiaoheng Li $^{1}{\text { Zhangli } \mathrm{Hu}^{2 * *}}^{2 *}$
}

${ }^{1}$ Shenzhen Centre for Diseases Control and Prevention, Shenzhen, China; *They contributed equally to this work;

${ }^{2}$ College of Life Science, Shenzhen University, Shenzhen, China; **Corresponding Author.

Email: huzl@szu.edu.cn

Received 30 November 2009; revised 5 January 2010; accepted 15 January 2010.

\begin{abstract}
To study immune reactions and the mechanism of anergy induced by recombinant enterotoxin A (rSEA) of Staphylococcus aureus. The gene encoding SEA was cloned from standard strain of $S$. aureus and high efficiently expressed in $E$. coli. After immunization with purified rSEA, mice were examined for production of specific antibody, subtype of IgG, cytokine mRNA levels such as IFN- $\gamma$, IL-2 secretion and T-cell surface PD-1 expression. Results showed that high levels of specific antibodies were produced in two weeks of primary immunization shot. During this time, humoral immune reactions prevailed $\left(\mathrm{IgG}_{2 \mathrm{a}} /\right.$ $\left.\mathrm{IgG}_{1}<1\right)$. During the early phase, Th1 type cytokine mRNA is expressed at a higher level than Th2 type, indicating cellular immune reaction prevailed. Splenocyte IFN- $\gamma$ secretion was significantly decreased after boosting immunization. The PD-1 expression was detected by a flow cytometry examination in the surface of T- lymphocytes which were induced by rSEA, and the expression of PD-1 molecules increased along with the number of boosting and the time after immunization.
\end{abstract}

Keywords: Staphylococcus Aureus; Enterotoxin A; Immune Respose

\section{INTRODUCTION}

Superantigens are distinguished from ordinary antigens by the ability that activates multiple T-cell clones. A very small amount can very effectively initiate the activation of immune system. Thus, superantigens have been considered to be widely applicable in tumor immune therapy. Their efficacy has also been considered superior to exogenous cell factor. With the development of relevant theory, superantigens have attracted much attention of studies [1,2]. Earlier studies applied superantigens independently in anticancer therapy. Later, they were used after modification with targeted monoclonal antibody, or as an enhancer of tumor vaccine, or gene vaccine or in combination with other therapy. In the United States, a superantigen Fab-SEA has been tested for anti-cancer ability in phase I clinical studies. In China, superantigens have been used to promoted leukocytes $[3,4]$.

Most currently known exogenous superantigens are toxins from bacteria. For example, the antigen studied in the currently report, enterotoxin A of Staphylococcus aureus, is one of these toxins. Superantigens that produced by bacteria upon infection may cause shock, fever, dehydration, skin eruption, organ failure, even death. The pathogenesis is that toxin superantigens stimulate large number of T-cells to proliferate, induce the secretion of cytokines from T-cell and APC cell, which result in immune system disorder. Superantigens induced disease may occur through several ways: 1) Superantigen is the directly cause of the disease, such as toxic shock and food intoxication. 2) Superantigen enhances the effect of the other infectious factors. 3) Superantigen induces autoimmune reaction by activating large number of $\mathrm{T}$ and B cells [5]. Up to date, there is no proof on the relationship between superantigen and some disease. However, the effects of superantigens in food intoxication, toxic shock and some infectious disease are very clear [6]. An understanding of the mechanism by which bacterial superantigens activate $\mathrm{T}$-cell and pathogenesis is theoretical bases for the application of superantigens in cancer immunotherapy. To this end, we studied the humoral and cellular immune reactions to recombinant SEA protein (rSEA), explored the relationship between inhibitory lymphocyte receptors and anergy induced by rSEA.

\section{EXPERIMENTS}

\subsection{Materials and Methods}

2.1.1. Materials

Staphylococcus aureus was provided by Shenzhen Cen- 
tre for Diseases Control and Prevention. Two strains of $E$. coli, DH5 $\alpha$, BL21(DE3) and pET-28a were the collection in the author's laboratory. Cloning vector pGEM-T easy was purchased from TaKaRa.

Antibody FITC-CD3, PE-PD-1 and corresponding negative control were purchased from BD Company. ELISA kit was purchased from Shenzhen Biotech Limited. Trizol and reverse transcription kit were purchased from TaKaRa Bio Inc. 1640 media, Lympho-Spot TM serum-free media and mice IFN- $\gamma$ ELISPOT kit were purchased from Dakewei Biotech Ltd. One hundred and eight BALB/c mice were purchased from Guangdong Center of Experimental Animal, maintained according to standard clean protocol. The mice used in the experiment were 15-20 g of body weight, 6-8 weeks of age.

\subsubsection{Preparation of rSEA}

Primers were derived from GenBank SEA sequence (AY827552): sea F: 5'GCC GCT AGC ATG AAA AAA ACA GCA TTT ACA TTA C 3' (underlined is NheI digestion site); sea R: 5'CGC CGT CGA CTT AAC TTG TAT ATA AAT ATA TAT CAA 3' (underlined is Sal I digestion site).

DNA template was from SEA producing standard strain. PCR product was prepared with sea $F$ and sea $R$ primers, separated with $1.5 \%$ agarose gel electrophoresis. After purification, the PCR product was inserted in pGEM-T easy vector. The recombinant plasmid was propagated in DH $5 \alpha$, selected with ampicillin on LB plate.

An expression vector of SEA was constructed by subcloning of the gene in pET-28a plasmid with E. coli BL21 (DE3) host. A recombinant clone was cultured in shaking incubator and induced by IPTG (final concentration $1 \mathrm{mmol} / \mathrm{L}$ ) for 6 hours. Bacteria were centrifuged, lysed with ultrasound. After centrifugation, rSEA was in the pellet. Subsequently, the pellet was resuspended in $8.0 \mathrm{~mol} / \mathrm{L}$ urea, and purified by $\mathrm{Ni}^{2+}$ affinity chromatography. The elution solution was imidazole $(500 \mathrm{mmol} / \mathrm{L})$. The rSEA protein was refolded and further purified to a high purity.

\subsubsection{IgG Level Determination by ELISA Assay}

Twenty BALB/c mice were assigned to either experiment group or control group at random. Lyophilized rSEA was resuspended in PBS and diluted to targeted concentration, mixed with equal volume of adjuvant, and injected subcutaneously into mice. The first injection was $100 \mu \mathrm{g}$, the second and third injection was 50 $\mu \mathrm{g}$ each at 2 weeks interval. Tail blood was collected each week before and after injection for 8 continuous weeks.

rSEA protein was diluted in embedding buffer to $10 \mu \mathrm{g} / \mathrm{mL}$, aliquoted to multi-well plate (100 mL/well).
The plate was incubated at $4{ }^{\circ} \mathrm{C}$ overnight, and then washed with PBST (PBST containing 0.05\% Tween-200) three times, blocked with blocking solution for $1 \mathrm{~h}$ at $37^{\circ} \mathrm{C}$. Subsequently, serum from each experiment groups were diluted 1:1000 in blocking solution, added to the plate, incubated at $37^{\circ} \mathrm{C}$ for 1 hour. The plated was then washed with PBST for 3 times. HRP conjugated Rabbit anti-mouse IgG (1:5000) was added to the plate and incubated for 1 hour at $37^{\circ} \mathrm{C}$, washed with PBST three times. Finally, diaminobenzene substrate solution containing hydrogen peroxide was added to the plate and allowed to develop for $10 \mathrm{~min}$ in dark. The reaction was stopped by addition of $2 \mathrm{~mol} / \mathrm{L} \mathrm{H}_{2} \mathrm{SO}_{4}$. Absorbance was determined with spectrometry at $450 \mathrm{~nm}$.

\subsubsection{Subtype Antibody Level Determination with ELISA}

Serum antibody subtypes were determined at 3,5 and 7 weeks after immunization. The method was the same as described above, except the secondary antibody was replaced by HRP conjugated mice $\operatorname{IgG}_{1}(1: 1000)$ and mouse $\operatorname{IgG}_{2 \mathrm{a}}(100 \mu \mathrm{L} / \mathrm{mL})$.

\subsubsection{RT-PCR Analysis of Spleen Cytokine Expression}

Sixty BALB/c mice were assigned to two groups and immunized with rSEA. Splenocytes were collected from 5 mice in each group at $0 \mathrm{~h}, 2 \mathrm{~h}, 12 \mathrm{~h}, 24 \mathrm{~h}, 2 \mathrm{w}$ and $3 \mathrm{w}$ post immunizations. Spleen total RNA was extracted with Trizol according to manufacturer's instruction. The mRNA levels of IL-2, IL-4 were determined with RT-PCR using specific primers. IL-2 primers were 5'- CTT CAA GCT CCA CTT CAA GCT-3' (forward) and 5'-CCA TCT CCT CAG AAA GTC CAC C-3' (reverse). The amplicon was 198 bp. IL-4 primers were 5'-CAT CGG CAT TTT GAA CGA GGT CA-3' (forward) and 5'-CTT ATC GAT GAA TCC AGG CAT CG-3' (reverse). The IL-4 amplicon was 203 bp. The expression of $\beta$-actin was used as internal control. The primers for $\beta$-actin were 5'-CAT CCG TAA AGA CCT CTA TGC CAA C-3' (forward) and 5'-ATG GAG CCA CCG ATC CAC A-3' (reverse). The $\beta$-actin amplicon was $238 \mathrm{bp}$. The thermal cycles of PCR were: pre denaturation at $94^{\circ} \mathrm{C}$ for $5 \mathrm{~min}, 30$ cycles of amplification $\left(94^{\circ} \mathrm{C} 30 \mathrm{~s}, 55^{\circ} \mathrm{C} 30 \mathrm{~s}\right.$ and $72^{\circ} \mathrm{C}$ for $\left.1 \mathrm{~min}\right)$. RT-PCR product was analyzed by $1 \%$ agarose gel electrophoresis [7].

2.1.6. Relative Quantification of Cytokine Expression Gel image was scanned with UVI system, and bands were quantified with UV band software. Band intensities were determined using $\beta$-actin as internal control. The relative expression levels (Ling, R.Y., et al.,) of cytokines were determined by:

Relative expression level $=$ (test gene band inten- 
sity $) /(\beta$-actin band intensity $) \times 100 \%$.

\subsubsection{IFN- $\gamma$ Detection by ELISPOT Assay}

Twenty four mice were assigned to either control group, or single immunization group or boost group $(n=8)$, injected intraperitoneally with rSEA (100 $\mu \mathrm{g} / \mathrm{animal})$ or PBS (control group). Mice were sacrificed by cervical dislocation $24 \mathrm{~h}$ after the last injection, and splenocytes were isolated with sterile procedure.

Lymphocyte preparation: spleen tissue was pressed against 200 micron mesh, filtered and spun. The second layer low density cells were collected and washed with 1640 medium. Cells were resuspended in Lympho-Spot TM serum-free medium. Cell concentration was adjusted to $2 \times 10^{6} / \mathrm{mL}$ and examined with trypan blue exclusion assay. Cell viability was greater than $95 \%$.

IFN- $\gamma$ detection with ELISPOT pre-embedded kit: 1$)$ plates were seeded with splenocytes $\left(1 \times 10^{5}\right.$ cells/well $)$, which were stimulated with $5 \mu \mathrm{g} / \mathrm{mL}$ rSEA antigen. Each sample was done with triplicates. ConA $(5 \mu \mathrm{g} / \mathrm{mL})$ or medium was added to the positive or negative control wells respectively. Cells were cultured at $37^{\circ} \mathrm{C}$ for $36 \mathrm{~h}$. After wash, biotinated anti mouse IFN- $\gamma$ was added to the wells $(100 \mu \mathrm{L} /$ well $)$, and the plate was incubated for $1 \mathrm{~h}$ at room temperature. 2) After washed, streptoavidinHRP was added to each well $(100 \mu \mathrm{L} /$ well $)$ and incubated for $1 \mathrm{~h}$ at room temperature. 3) Upon wash, HRP substrate AEC was added to the plate. Color was developed in dark. The plate was washed with water and dried. 4) Spots were counted. The unit was defined as spots $/ 10^{5}$ spleen cells. Negative control has less than 10 spots $/ 10^{5}$ splenocytes. Test wells that had greater than 2-fold the spot number of the negative control well was counted as positive.

\subsubsection{PD-1 Expression Determination by Flow Cytometry}

Sixty mice were assigned to either single immunization group or boost group or control group $(n=20)$ and injected with rSEA or PBS. Animals were sacrificed at $2 \mathrm{~h}$ or $24 \mathrm{~h}$ post last immunization. Splenocytes were isolated as described above. Cells were stained with FITC- conjugated anti-CD3 and PE-labeled PD-1. After fixation, cells were examined with flow cytometer. Cell concentration was adjusted to $2 \times 10^{6}$ cells $/ \mathrm{mL}$. $500 \mu \mathrm{L}$ of the cell suspension was loaded to each of two flow tubes. FITC Anti-Mouse CD3 (5 $\mu \mathrm{L})$, PE Anti-Mouse PD-1 (5 $\mu \mathrm{L})$ or equal volume of respective control solution was added to the tubes. After mix by shaking, the tubes were set in dark for $45 \mathrm{~min}$ at room temperature. Cells were spun, washed with PBS once and resuspended in $200 \mu \mathrm{L}$ PBS, fixed with 500 $\mu \mathrm{L}$ paraformaldehyde $(4 \%)$ and examined with flow cytometry.

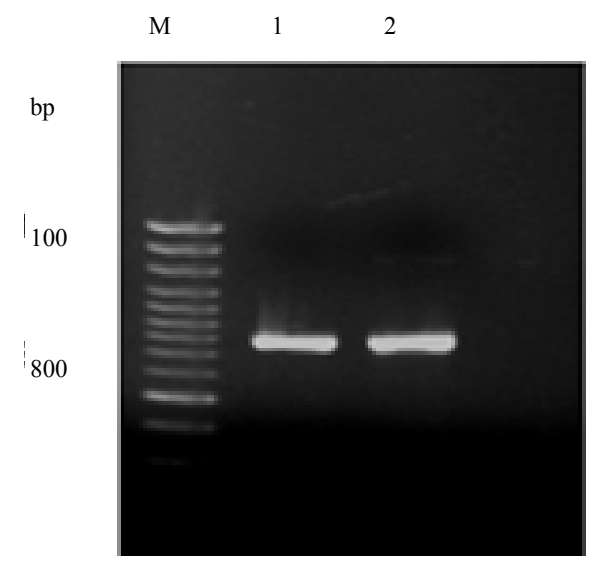

Figure 1. Amplification of SEA gene from Staphylococcus aureus genomic DNA. M was 100 bp DNA Ladder, lane 1, 2 was PCR product of SEA gene.

\subsubsection{Statistical Analysis}

Data were analyzed with SPSS 11.5 software. Results were presented as mean \pm s. Comparisons between groups were analyzed with ANOVA. Statistical significances were inferred when $p<0.05$ or $p<0.01$.

\section{RESULTS AND DISCUSSION}

\subsection{Cloning of SEA Gene}

With the specific primers, we identified 2 strains, out of 10 wild type $S$. aureus, to be positive for producing enterotoxin A (Figure 1). The PCR product was inserted into pGEM-T easy vector and propagated in DH5 $\alpha$. The plasmid insert was sequenced, which confirmed that the insert sequence was identical to AY827552. The size was 786 bp encoding 261 residues.

\subsection{Expression, Purification and Refolding of rSEA}

The recombinant plasmid pET-28-SEA was transformed into $E$. coli BL21 cells and the transformants were induced with IPTG for $6 \mathrm{~h}$. Bacterial cells were disrupted with ultrasound. Upon centrifugation, the recombinant protein was identified in the precipitate, demonstrating that rSEA was expressed as inclusion body. During $\mathrm{Ni}^{2+}$ affinity chromatography, rSEA was eluted at $500 \mathrm{mmol} / \mathrm{L}$ imidazole. After purification and refolding, high purity rSEA was obtained (Figure 2(a)).

Immunobloting analysis showed that the refolded SEA was reactive with specific polyclonal antibody and the molecular weight was as expected $31 \mathrm{kD}$. This result demonstrated that the recombinant SEA has similar antigenicity as the natural one (Figure 2(b)).

\subsection{Humoral Immunity Induced by rSEA}

In order to study the process of humoral immunity induced by SEA, we examined specific IgG production in sera from mice immunized three times with conventional 


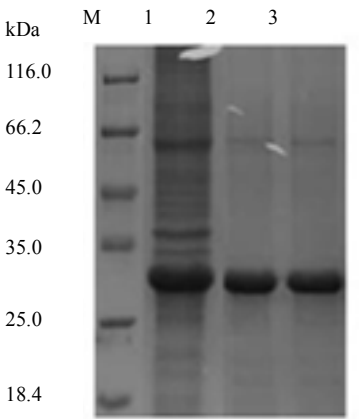

(a)

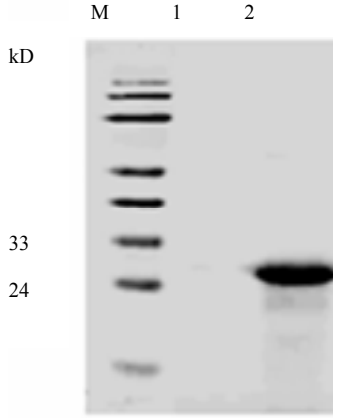

(b)
Figure 2. (a) SDS-PAGE analysis of recombinant SEA(rSEA) preparation by affinity chromatography. $\mathrm{M}$ was molecular weight markers, lane 1 was cell extract from pET28a-rSEA transformed E.coli, Lane 2,3 was recombinant SEA of affinity chromatography; (b) Western-blot analysis of purified rSEA protein. $\mathrm{M}$ was molecular weight markers, lane 1 was negative sera act as control sera, lane 2 was anti-rSEA sera act as first antibody.

protocol. Results showed that specific antibody level in the immunized mice $\left(\mathrm{OD}_{450}=2.492 \pm 0.082\right)$ was markedly higher than that in the control mice $\left(\mathrm{OD}_{450}=0.054\right.$ $\pm 0.032)$ three weeks after the first immune shot $(\mathrm{P}<$ $0.01)$. There were no significant changes in antibody levels before and after the second (week 3,4) and third shots (week 5,6) in the immunized mice $(\mathrm{P}>0.05)$. Two weeks after the last shot, the antibody level was slightly reduced, but not significantly $(\mathrm{P}>0.05)$.

To evaluate the immunogenicity of rSEA as a candidate of cancer therapeutics, we assessed specific serum antibody in immunized mice, investigated the process of rSEA induced humoral immunization. The key steps of humoral immunization are the activation and proliferation of B-lymphocytes, which require the stimulation of secreted factor or exogenous antigen and the assistance of $\mathrm{CD}^{+} \mathrm{T}$ cells. We performed ELISA analysis on the mouse sera collected at different time point, discovered that specific immunoreactions were strongly induced within two weeks of immunization. (The immunized animals produced significantly higher antibody levels than the control animals and) the high antibody levels were maintained for a long time. Compared with a single shot immunization, boost immunization did not increase rSEA specific antibody levels (Specific IgG induced by rSEA can be used to destroy cancer cells through the activation of complement and superantigen-dependent cell-mediated cytotoxicity) [7].

\subsection{IgG Subtype Induced by rSEA}

Changes in specific IgG subtype levels were shown in Figure $3 . \mathrm{IgG}_{1}$ levels were significantly different between control group ( $\mathrm{C}$ group) and test group ( $\mathrm{T}$ group) three weeks after immunization $(\mathrm{P}<0.05)$. The differences were even more pronounced 5 and 7 weeks after immunization $(\mathrm{P}<0.01)$. $\operatorname{IgG}_{2 \mathrm{a}}$ levels were different between the two groups only at three weeks after immunization $(\mathrm{P}<$ $0.05)$. In the test group, specific $\operatorname{IgG}_{1}$ levels continuously increased along with time and the number of immune shots (Figure 3(a)). In contrast, $\operatorname{IgG}_{2 \mathrm{a}}$ levels decreased during the same period (Figure $3(\mathbf{b})$ ). The ratio of $\mathrm{IgG}_{2 \mathrm{a}} / \mathrm{IgG}_{1}$ did not change in the control mice at 3, 5 and 7 weeks (data not shown). However, this ratio showed a trend of decrease in the test group (Figure 3(c)), which

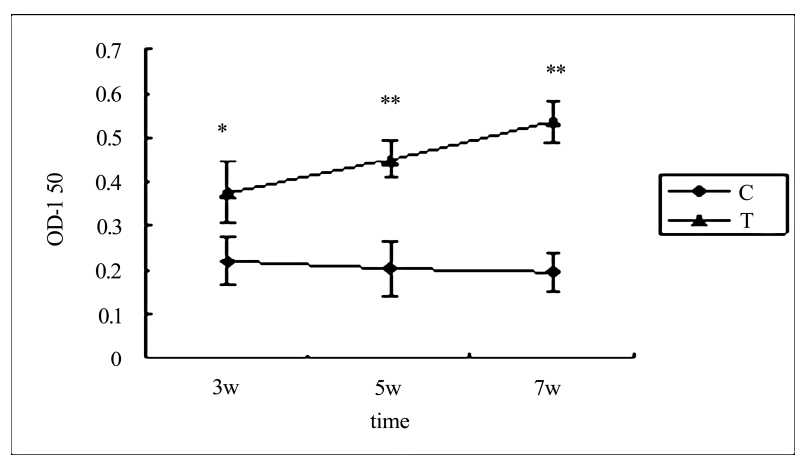

(a)

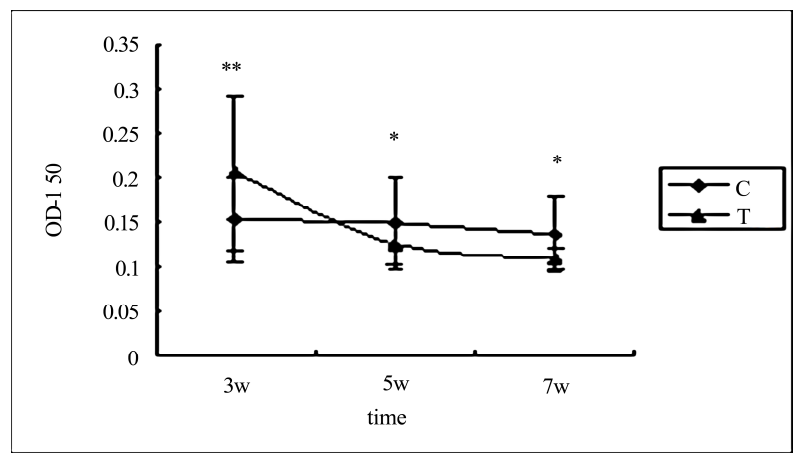

(b)

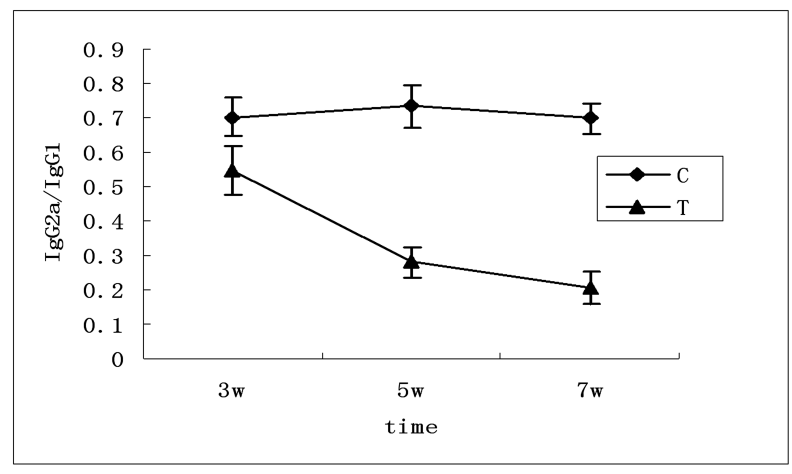

(c)

Figure 3. Specific IgG subtypes induced by rSEA from mice immunized. (a) $\operatorname{IgG}_{1}$; (b) $\operatorname{IgG}_{2 \mathrm{a}}$; (c) $\operatorname{IgG}_{2 \mathrm{a}} / \mathrm{IgG}_{1}$ 
was significantly different from that of control group at 5 and 7 weeks $(\mathrm{P}<0.05)$.

$\mathrm{IgG}_{1}(\mathrm{Th} 2)$ and $\mathrm{IgG}_{2 \mathrm{a}}$ (Thl) are type markers of immunoreactions. The ratio of $\operatorname{IgG}_{2 \mathrm{a}} / \mathrm{IgG}_{1}$ indicates whether the humoral immunoreactions are dominated by Th1 or Th2. In the current study, we selected peripheral serum from mice two weeks after immunization, assessed levels of $\operatorname{IgG}_{1}$ and $\operatorname{IgG}_{2 \mathrm{a}}$ with ELISA. Results showed that $\mathrm{IgG}_{1}$ level was higher than $\mathrm{IgG}_{2 \mathrm{a}}$ level after initial immunization, and that $\operatorname{IgG}_{1}$ exhibited a trend of increase along with time and the increase in the number of shots (Figure 4(a)), while $\operatorname{IgG}_{2 \mathrm{a}}$ level showed a trend of decrease (Figure 4(b)). Thus, the ratio of $\operatorname{IgG}_{2 \mathrm{a}} / \mathrm{IgG}_{1}$ was always smaller than 1 , and exhibited a trend of decline (Figure 4(c)). These data indicated that rSEA-induced immunity was dominated by $\mathrm{Th} 2$ reaction and the dominance tends to be enhanced by boost shots.

\subsection{Effects of rSEA on Splenocyte Cytokine mRNA Levels}

Messenger RNA levels of IFN- $\gamma$, IL-4 and $\beta$-actin in

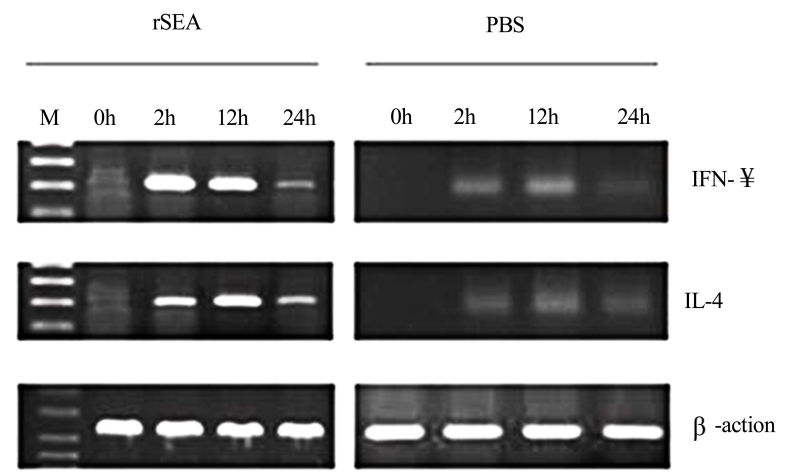

(a)

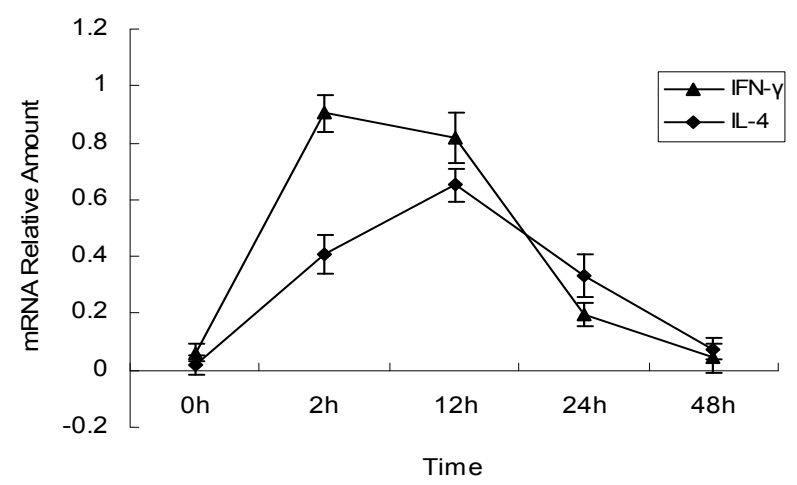

(b)

Figure 4. (a) RT-PCR results of cytokine in spleen of immunized mice with rSEA, PBS acted as control of immunization, and fragment of $\beta$-action was as a RT-PCR control; (b) Analysis of Cytokine mRNA expression in spleens of immunized mice at different time. splenocytes were analyzed with RT-PCR. The PCR products were the expected sizes $198 \mathrm{bp}, 203 \mathrm{bp}$ and 238 bp (Figure 4(a)). Before immunization (0 h), IFN- $\gamma$, IL-4 mRNA was not detectable. After immunization, mRNA levels increased. Levels of mRNA in immunized groups were significantly higher than that in PBS group at 2,12 and $24 \mathrm{~h}(\mathrm{P}<0.01)$. There was no significant difference between the two groups in the mRNA levels $24 \mathrm{~h}$ after immunization. At 2 and $12 \mathrm{~h}$ after immunization, IFN- $\gamma$ mRNA levels were higher than IL-4 in the immunized grouping $(\mathrm{P}<0.01)$. In contrast, at $24 \mathrm{~h}$ after immunization, IL-4 mRNA level was higher than IFN- $\gamma$ mRNA $(\mathrm{P}<0.05)$ (Figure 4(b)).

We investigated mRNA levels of two cytokines after immunization with semi-quantitative RT-PCR. Results showed that both cytokines were greatly increased after induction with rSEA. At $2 \mathrm{~h}$ post immunization, IL-2 mRNA was higher than IL-4 mRNA level and reduced soon after. In contrast, IL-4 mRNA was low at $2 \mathrm{~h}$ after immunization and gradually increased, peaked at $12 \mathrm{~h}$ and then gradually decreased. Therefore, during immune reaction, Th1 type cytokine proliferated earlier than Th2 type cytokine, but the level rapidly reduces to become lower than Th2 cytokine level. This kinetic process is consistent with the change in serum IgG subtype $[8,9]$. Microphages $(\mathrm{M} \Phi)$ are very important immune cells with unique anti-cancer effect. They provide immune surveillance, antigen presentation and effector functions. Through antigen presentation, $\mathrm{M} \Phi$ activates T-cell and enhances specific anti-cancer immunity. $M \Phi$ also nonspecifically destroys cancer cell upon contact. Activated $\mathrm{M} \Phi$ releases many cytokines and bioactive factors that regulate cancer immunity [10]. IFN- $\gamma$ secreted by T cells and NK cells is the most potent $\mathrm{M} \Phi$ activator. However, IFN- $\gamma$ production during tumor genesis is insufficient, leading to insufficient $M \Phi$ activation. Superantigens have powerful immune activation ability that can induce the release of large amount of cytokines. This point has been proven by previous experiments $[11,12]$. To further study the relationship between rSEA and IFN- $\gamma$ secretion, we analyzed splenocyte IFN- $\gamma$ secretion in immunized mice with ELISPOT technique. Our results showed that splenocyte can secret IFN- $\gamma$ at high frequency after primary immunization, (which is significantly different from the control splenocytes). This cellular immune reaction is rSEA specific, because splenocyte stimulated by other antigens produced little IFN- $\gamma$. Boost shot did not enhance antibody production $(\mathrm{P}>0.05)$, suggesting rSEA as a superantigen may induce anergy after primary immunization [13].

\subsection{Effects of rSEA on Splenocyte IFN- $\gamma$}

To further explore immunoreactions to rSEA, we as sessed 
changes in IFN- $\gamma$ secretion by splenocytes with ELISPOT assay. As shown in Figure 5, little specific IFN- $\gamma$ were produced without stimulators. In contrast, IFN- $\gamma$ was produced in the three groups of mice stimulated with rSEA or ConA. Mice in single shot group and boosted group produced more IFN- $\gamma$ than that in control group $(\mathrm{P}<0.01)$. There were no differences in IFN- $\gamma$ levels between the single shot group and the boost group $(\mathrm{P}>0.05)$.

Regulation of immunity includes initiation and termination of immune reactions, dependent of internal and external signal. External signal is transmitted through surface receptors. There are stimulatory and inhibitory receptors that positively or negatively regulate cell activation. Inhibitory receptors of the B7 family include cytotoxic T lymphocyte associated antigen 4 (CTLA-4), programmed death-1 (PD-1) and $\mathrm{B}$ and $\mathrm{T}$ lymphocyte attenuator (BTLA) [14]. These three inhibitory receptors belong to the $\mathrm{CD} 28$ family. Upon binding with different members of B7 family, these receptors can inhibit activa-

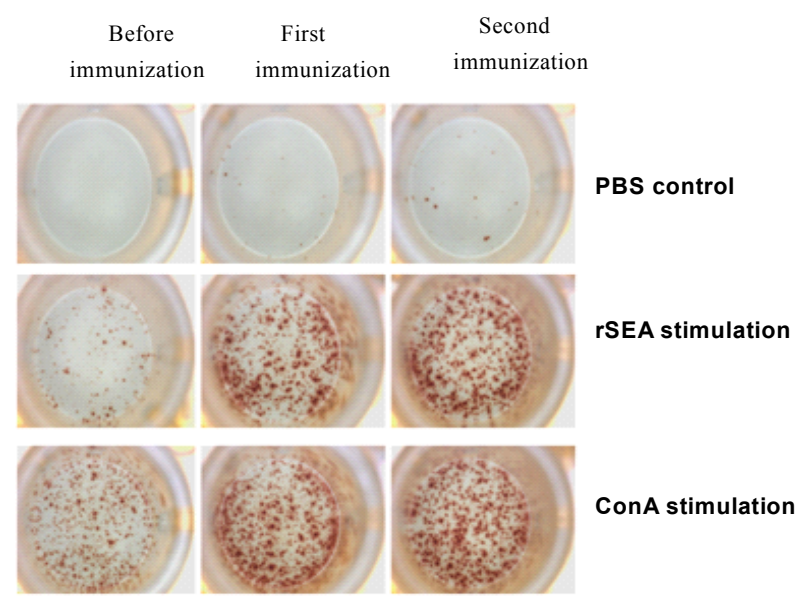

Figure 5. ELISPOT analysis of IFN- $\gamma$ in mice spleen immunized with rSEA at different stage of immunization. tion of immune reactions. The mechanism has been widely investigated. Studies have shown that interaction between PD-1 and its complementary PD-L leads to the inhibition of T-cell proliferation and the attenuation of IL-2, -10 and IFN- $\gamma$ secretion. This mechanism is very important for organ transplant, autoimmune disease and cancer immunity. An examination of splenocyte IFN- $\gamma$ secretion with ELISPOT assay revealed that boost shot did not enhance cellular immunity, suggesting possibility of anergy after rSEA immunization.

\subsection{T-Cell PD-1 Expression Induced by rSEA}

PD-1 expression in T-cell was examined $2 \mathrm{~h}$ and $24 \mathrm{~h}$ after a single shot immunization or boost immunization. Splenocytes were stained with FITC-anti-CD3 and PEanti-PD-1, fixed and examined with flow cytometer. Results showed that T-cell surface PD-1 expression in the rSEA immunized mice were significantly higher than that in the control mice $(\mathrm{P}<0.01)$. Significant differences were observed between $2 \mathrm{~h}$ and $24 \mathrm{~h}$ in the expression of PD-1 in the single shot and boost shot immunization. Also, significant differences were also observed in PD-1 expression between single shot and boost shot $(\mathrm{P}<$ 0.91 ) at $2 \mathrm{~h}$ and $24 \mathrm{~h}$ (Figure 6).

We examined the expression of PD-1 in immunized splenocytes with flow cytometry to interrogate its relationship with immunized time and the number of shots. Results showed rSEA immunized mice had higher PD-1 expression and boost shots further enhanced PD-1 expression. PD-1 levels in boosted group were higher than that in the non-boosted group. Also PD-1 levels at $24 \mathrm{~h}$ after immunization were higher than that at $2 \mathrm{~h}$. This reaction was rSEA-specific, since the PD-1 level in control group did not change over time. These results explained unresponsiveness of IFN- $\gamma$ level to boost shots, suggesting that inhibitory receptor PD-1 attenuated T-cell proliferation and IFN- $\gamma$ secretion [15].
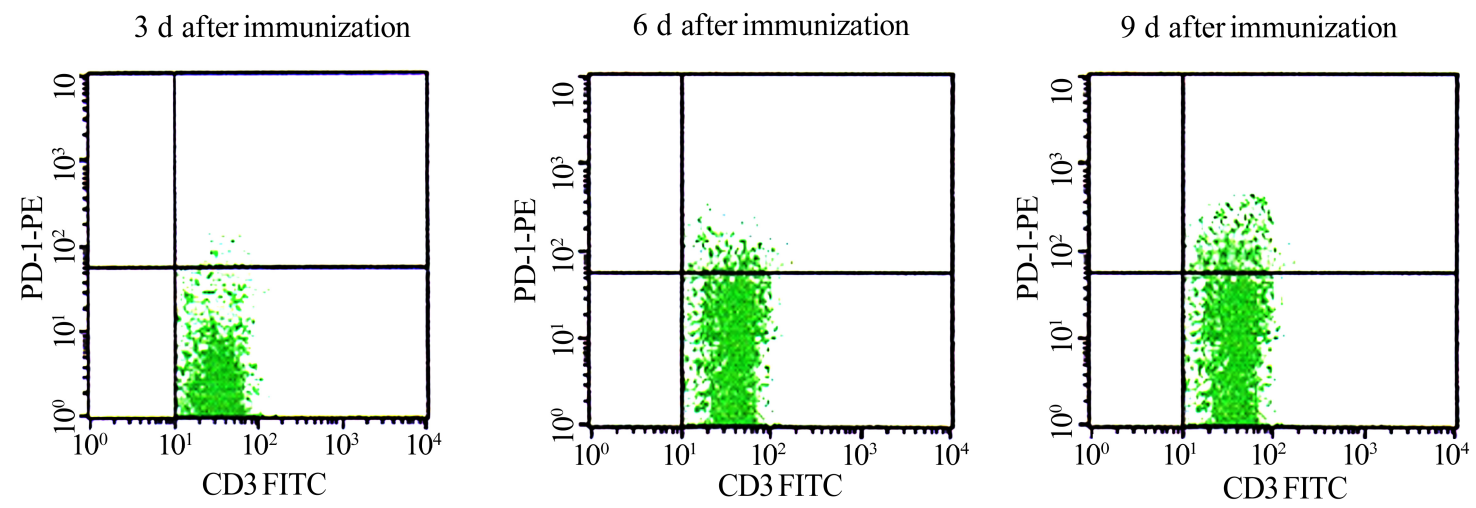

Figure 6. Expression of PD-1 molecular on spleen T cells of mice immunized with rSEA, FCM showed different expression of PD-1 molecular on 3d ,6d and 9 d.after immunized. 


\section{CONCLUSIONS}

Superantigens are powerful activator of T-cell. Distinct from ordinary antigens, superantigens can directly attach to the outer groove of MHC-II and V $\beta$ domain of TCR on T-cell surface, without the processing by antigen presentation cells. Even a trace amount of superantigen can activate $5 \%-20 \%$ of T-cells. Therefore, superantigens have been tried in cancer immune therapy to promote internal anticancer immunity. Good results have been obtained from these trials. However, it has also been found that superantigen may induce apoptosis and inability after activating T-cells, leading to the attenuation of response to additional stimulation. This property of tolerance induction limits the efficacy of superantigens. Therefore, we have used rSEA to immunized mice, studied the characteristics of rSEA induced specific humoral immunity and cellular immunity and the mechanism of anergy. These studies could provide foundations for further studies of superantigens as tumor suppressors.

rSEA has superantigen properties and that it can induce powerful humoral and cellular immune responses. However, boosting immunization with rSEA caused anergy through PD-1 mediated inhibition.

\section{ACKNOWLEDGEMENTS}

The authors acknowledge research funding from the National Natural Science Foundation of China (Grant No. 30770340,30470281 ), the national major program of Science and technology for water pollution control and restoration in china (Grant No. 2009ZX07423-003) and Shenzhen Grant Plan for Science and Technology.

\section{REFERENCES}

[1] Lu, S.Y., Sui, Y.F., Li, Z.S., et al. (2004) Superantigen-SEA gene modified tumor vaccine for hepatocellular carcinoma: An in vitro study. World Journal of Gastroenterology, 10(1), 53-57.

[2] Proft, T. and Fraser, J.D. (2003) Bacterial superantigens. Clinical and Experimental Immunology, 133(3), 299-306.

[3] Balaban, N. and Rasooly, A. (2000) Staphylococcal enterotoxins. International Journal of Food Microbiology, 61(1), 1-10.

[4] Cheng, J.D., Babb, J.S. and Langer, C. (2004) Individualized patient dosing in phase I clinical trials: The role of escalation with overdose control in PNU-214936. Journal of Clinical Oncology, 22(4), 602-609.

[5] Han, W.N., Cao, Y.X. and Li, Qi. (2004) The different influence of SEA on the direnfiafion and proliferation of $\mathrm{Tcl}$ and $\mathrm{Tc} 2$ subsets. Chinese Journal of Microbiology and Immunology, 24(4), 300-303.

[6] Petersson, K., Forsberg, G. and Walse, B. (2004) Interplay between superantigens and immunoreceptors. Scandinavian Journal of Immunology, 59(4), 345-355

[7] Melanie, J.R., Nisebita, S. and Avery, A. (2006) Differential regulation of cytokine production by CD1d-restricted NKT cells in response to superantigen staphylococcal enterotoxin B exposure. Infection and Immunity, 74(1), 282-288

[8] Mondal, T.K., Bhatta, D., Biswas, S. and Pal, P. (2002) Superantigen-induced apoptotic death of tumor cells is mediated by cytotoxic lymphocytes, cytokines, and nitric oxide. Biochemical and Biophysical Research Communications, 290(4), 1336-1342.

[9] Zhang, M.H., Cao, X.T. and Chen, G.Y. (1999) Presentation of tumor antigen to T lymphocytes by macrophage like bone marrow stromal cells. Chinese Journal of Microbiology and Immunology, 19(5), 412-416.

[10] Ling, R.Y., Ding, J.B. and Wen, H. (2003) A semi-quantitative RT-PCR analysis for IL-2/IL-4 mRNA expression in heart transplantation in rats. Journal of Xinjiang Medical University, 26(5), 427-429.

[11] Frauwirth, K.A., Alegre, M.L. and Thompson, C.B. (2000) Induction of T cell anergy in the absence of CTLA-4/B7 interaction. Journal of Immunology, 164, 2987-2993.

[12] Honstettre, A., Mège, J.L., Gérard, L., Aubaniac, J.M. and Drancourt, M. (2003) Relationship of relapsing hip prosthesis infection by Staphylococcus aureus with gamma interferon deficiency. Journal of Clinical Microbiology, 41(11), 5344-5346.

[13] Plaza, R., Rodriguez, J.L. and Juarez, C. (2007) Staphylococcal enterotoxin B in vivo modulates both gamma interferon receptor expression and ligand-induced activation of signal transducer and activator of transcription 1 in T cells. Infection and Immunity, 75(1), 306-313.

[14] Xu, G.L., Zhu, X.H., Guo, B. and Wu, Y.Z. (2004) Involvement of CTLA-4 in T-cell anergy induced by staphylococcal enterotoxin A in vitro. Molecular Immunology, 41(1), 1-8.

[15] Keir, M.E., Francisco, L.M. and Sharpe, A.H. (2007) PD-1 and its ligands in T-cell immunity. Current Opinion in Immunology, 19(3), 309-314. 